\title{
Van der Waals integration before and beyond two-dimensional materials
}

Yuan Liu ${ }^{1,2}$, Yu Huang1,3* \& Xiangfeng Duan ${ }^{3,4 *}$

\begin{abstract}
Material integration strategies, such as epitaxial growth, usually involve strong chemical bonds and are typically limited to materials with strict structure matching and processing compatibility. Van der Waals integration, in which pre-fabricated building blocks are physically assembled together through weak van der Waals interactions, offers an alternative bondfree integration strategy without lattice and processing limitations, as exemplified by two-dimensional van der Waals heterostructures. Here we review the development, challenges and opportunities of this emerging approach, generalizing it for flexible integration of diverse material systems beyond two dimensions, and discuss its potential for creating artificial heterostructures or superlattices beyond the reach of existing materials.
\end{abstract}

Semiconductor heterostructures and superlattices represent the essential material foundation for all modern electronics and optoelectronics. Integrating dissimilar materials with pristine interfaces is essential for creating functional devices by design and has

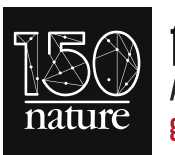
long been a pursuit of the materials science community. The current strategy for hetero-material integration generally relies on chemical epitaxial growth or physical vapour deposition (PVD). Chemical epitaxial growth methods, such as molecular beam epitaxy (MBE) and metal-organic chemical vapour deposition (MOCVD), have provided the highest-quality heterostructures for a wide range of state-of-the-art devices, including high-electron-mobility transistors, light-emitting diodes (LEDs) and quantum cascade lasers ${ }^{1}$. However, such integration relies on one-to-one chemical bonds and is usually limited to materials with highly similar lattice symmetry and lattice constants, and thus similar electronic properties, which often require similar processing conditions (Fig. la, b) ${ }^{2-4}$. Materials with substantially different lattice structures or processing conditions cannot be epitaxially grown together without generating too much interface disorder, which could severely alter their intrinsic properties (Fig. 1a, lower panel). For example, MBE involves strict constraints on the lattice constant or film thickness of the epitaxial layer, where the lattice mismatch $f$ should typically be smaller than $5 \%$ to prevent polycrystalline phase formation, and the epitaxial layer needs to be thinner than a critical thickness $d_{\mathrm{c}}$ (about $10 \mathrm{~nm}$ for $f=1 \%$ ) to prevent the formation of misfit dislocations ${ }^{5}$. Even with these requirements met, the resulting interface is still plagued by strain in both lattices, as can be observed in a typical two-dimensional electron gas superlattice ${ }^{6}$ (Fig. 1c). For thicker epitaxial layers, slight mismatching normally leads to misfit dislocations at the interface ${ }^{7}$ (Fig. 1d), which could propagate well beyond the interfaces and result in extensive threading dislocations when the mismatch increases ${ }^{8}$ (Fig. 1e). These constraints imposed by MBE are also valid for other chemical integration techniques, such as MOCVD or atomic layer deposition (ALD). For example, owing to substantial lattice structure differences, a high-quality dielectric thin film cannot be easily integrated on top of a crystalline semiconductor with pristine surface (for example, silicon or graphene). Such striking lattice differences often result in island formation during the nucleation stage and prevent uniform thin-film deposition (Fig. 1f) $)^{9,10}$.
On the other hand, conventional physical vapour deposition relying on high-energy vaporization of precursor materials is more flexible on material types and lattice structures, and is thus widely used for integrating highly disparate materials to create functional interfaces. However, the deposited materials are typically amorphous or polycrystalline and the resulting heterostructure interfaces are usually plagued by unavoidable deposition-induced defects and rich chemical disorders that dictate the interface properties. For example, vacuum evaporation and sputtering are standard processes used for depositing metal contacts on various semiconductors. Although widely used, such high-energy integration processes involve continuous bombardment of the contact region by high-energy metal atoms and clusters and strong local heating, typically producing a disordered glassy layer at the metal/ semiconductor interface $^{11}$ (Fig. 1g) and leading to the Fermi level pinning (FLP) effect and incontrollable Schottky barriers in semiconductor devices $^{12}$.

\section{Van der Waals integration}

Alternatively, van der Waals (vdW) integration, in which pre-fabricated building blocks are physically assembled together through weak vdW interactions, offers an alternative low-energy material-integration approach. Such a physical assembly method does not rely on oneto-one chemical bonds and does not involve direct chemical processing on existing materials; thus, it is not limited to materials that have similar lattice structures or require compatible synthetic conditions. It has therefore attracted considerable interest for integrating diverse two-dimensional (2D) atomic crystals with highly distinct lattice structures yet little chemical disorder at the interface ${ }^{13-16}$ (Fig. 2a). Although $\mathrm{vdW}$ integration has been well recognized and intensively explored in 2D devices, its applicability to other material systems (beyond 2D materials) and its prospects for scalable integration and practical applications have not been fully appreciated.

In principle, without the requirements of lattice matching and processing compatibility, such a bond-free integration strategy is not limited to a particular material dimension and could be generally applicable for flexible integration of radically different materials with distinct crystal structures (crystallinity, lattice symmetry, lattice constant), electronic properties (metals, semiconductors, insulators 
a
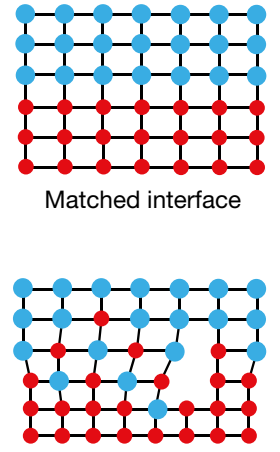

Mismatched interface

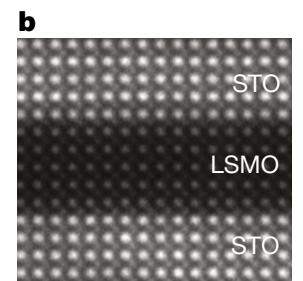

e

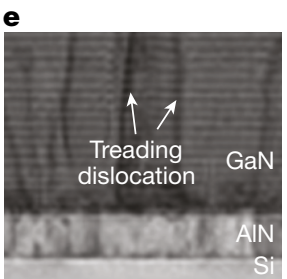

Fig. 1 | Structural characteristics of conventional bonded heterostructure interfaces. a, Schematic illustrations of bonded heterostructure interfaces: a lattice-matched interface (top) and a latticemismatched interface (bottom). b, Cross-sectional image of a latticematched interface (STO/LSMO; STO, $\mathrm{SrTiO}_{3} ; \mathrm{LSMO}, \mathrm{La}_{0.7} \mathrm{Sr}_{0.3} \mathrm{MnO}_{3}$ ) grown using pulsed laser deposition, with low interfacial strain and one-to-one chemical bonds. Image adapted from ref. ${ }^{4}$ with permission from PNAS. c, Cross-sectional image of InAs/AlSb interfaces grown using MBE, with clear interface strains. Image adapted from ref. ${ }^{6}$ with the permission of AIP Publishing. d, Cross-sectional image of a III-Sb/ $\mathrm{Si}$ interface grown using MBE. Misfit dislocations are observed owning

and superconductors) or material dimensions ${ }^{17-20}$ (zero-dimensional, 0D; one-dimensional, 1D; 2D; and three-dimensional, 3D) (Fig. 2b). Without direct chemical bonding (Fig. 2c), vdW integration could enable the creation of a series of artificial heterostructures and superlattices with atomically clean and electronically sharp interfaces between highly disparate materials, as exemplified in various vdW interfaces demonstrated recently (for example, $\mathrm{Bi}_{2} \mathrm{Te}_{3} / \mathrm{FeTe}$, graphene $/ \mathrm{Al}_{2} \mathrm{O}_{3}, \mathrm{MoS}_{2} / \mathrm{Au}$, phosphorene/molecules) ${ }^{15,18-20}$ (Fig. 2d-g).

In this Perspective, we start with the fundamental concepts of the $v d W$ distance, $v d W$ interaction and $v d W$ gap to generalize the definitions and requirements for $\mathrm{vdW}$ integration, and summarize current vdW building blocks. Next, we outline the historical background and highlight state-of-the-art vdW heterostructures with various material dimensions, including $1 \mathrm{D} / 1 \mathrm{D}, 0 \mathrm{D} / 2 \mathrm{D}, 1 \mathrm{D} / 2 \mathrm{D}, 2 \mathrm{D} / 2 \mathrm{D}, 2 \mathrm{D} / 3 \mathrm{D}$ and $3 \mathrm{D} / 3 \mathrm{D}$, and then discuss potential opportunities and challenges arising in these defect-free and pinning-free vdW interfaces. Overall, we hope to highlight the bond-free vdW integration as a general low-energy integration approach (as opposed to conventional high-energy chemical integration) that can impart unparalleled freedom to integrate distinct materials beyond the limits of traditional integration approaches, opening up new opportunities for fundamental studies and enabling unprecedented device functions and performance beyond the reach of existing materials.

\section{Bonded versus vdW-gapped interfaces}

The vdW interaction ${ }^{21,22}$, named after Dutch scientist Johannes Diderik van der Waals, generally includes three different types of intermolecular interactions: dipole-dipole interaction (Keesom force), dipole-induced dipole interaction (Debye force) and instantaneous dipole-induced dipole interaction (London force) (Fig. 3a). The vdW interaction has been simply modelled and approximated using a Lennard-Jones potential $^{23,24}$, which dates back to 1924 , with the lowest potential at an equilibrium centre-to-centre distance between two vdW-interacting atoms or molecules (for example, $0.38 \mathrm{~nm}$ for an argon dimer) (Fig. 3b) that is often referred to as the vdW distance $\left(d_{\mathrm{vdW}}\right)$ (Fig. 3c).

The vdW interaction strength is typically of the order of $0.1-10 \mathrm{~kJ}$ $\mathrm{mol}^{-1}$, about 2-3 orders of magnitude smaller than that of ionic or covalent bonds (about $100-1,000 \mathrm{~kJ} \mathrm{~mol}^{-1}$ ). Although being the weakest interaction, the vdW force $\left(>10^{-12} \mathrm{~N}\right.$ per atom pair or $\left.>10 \mathrm{~N} \mathrm{~cm}^{-2}\right)$
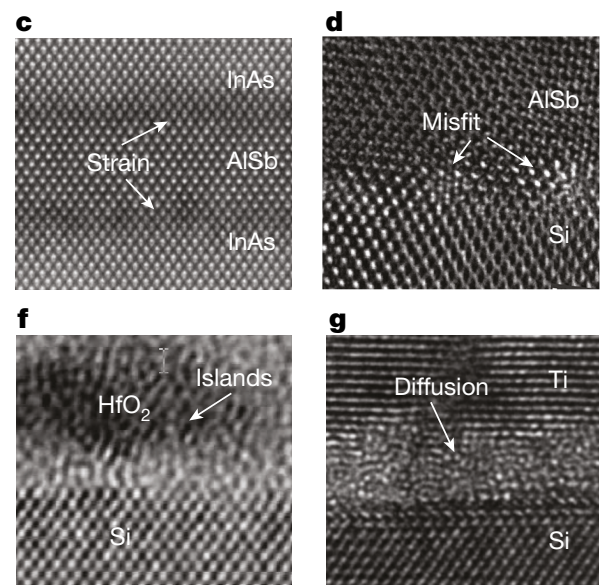

to the lattice mismatch. Image adapted from ref. ${ }^{7}$ with permission from Elsevier. e, Cross-sectional image of GaN(AlN)/AlN/Si superlattice grown by MOCVD. Threading dislocations are observed owing to large lattice mismatch. Image adapted from ref. ${ }^{8}$ with permission from IOP Publishing. f, Cross-sectional image of a $\mathrm{HfO}_{2} / \mathrm{Si}$ interface fabricated using ALD. Poor nucleation is observed on the $\mathrm{H}$-terminated silicon surface with discontinuous $\mathrm{HfO}_{2}$ islands. Image adapted from ref. ${ }^{9}$ with permission from Elsevier. $\mathbf{g}$, Cross-sectional image of Ti/Si interface fabricated using high vacuum deposition, with a glassy interfacial layer. Image adapted from ref. ${ }^{11}$ with permission from Elsevier.

within an intimate-contact interface is in fact much larger than the gravity force of typical integrated structure $\mathrm{s}^{25,26}$ and is strong enough to hold bulk materials (about $1-10 \mathrm{~N} \mathrm{~cm}^{-2}$ for 1-m-thick material) together against gravitational movement. Nonetheless, owing to their finite surface roughness, most interfaces between two rigid 3D bulk materials do not exhibit intimate contact to reach the vdW distance and fully activate the vdW interaction. Therefore, flexible low-dimensional materials or thin films are usually more likely to reach the vdW distance and activate the vdW interaction, and thus are more suitable for $\mathrm{vdW}$ integration.

The vdW gap, although frequently used in 2D materials research, does not have a clear definition and is often mixed with the 2D layer thickness or the 2D layer-to-layer distance. To ensure consistency and avoid confusion, here we define the vdW gap as the difference between the vdW distance and the covalent bond length (as labelled in Fig. 3c, d). Hence, the vdW gap $\left(g_{\mathrm{vdW}}\right)$ could be approximated using:

$$
g_{\mathrm{vdW}} \approx d_{\mathrm{vdW}}-r_{\mathrm{a}}-r_{\mathrm{b}}
$$

where $r_{\mathrm{a}}$ and $r_{\mathrm{b}}$ are the covalent radii of individual atoms. Using this equation, the $g_{\mathrm{vdW}}$ value of argon dimers can be calculated as $0.23 \mathrm{~nm}$. From this point of view, the vdW gap in layered materials is much smaller than their layer-to-layer spacing. As shown in Fig. 3d, the interlayer spacing of graphite is $0.34 \mathrm{~nm}$ and the calculated $g_{\mathrm{vdw}}$ is about $0.2 \mathrm{~nm}$ using equation (1). For other layered materials with different interlayer spacings ranging from about $0.3 \mathrm{~nm}$ to about $0.8 \mathrm{~nm}$ (Fig. 3e), the calculated $g_{\mathrm{vdW}}$ exhibits a similar value of around $0.2 \mathrm{~nm}$, comparable to that of argon dimer or graphite layers. Additionally, the $g_{\mathrm{vdW}}$ value between artificially assembled vdW interfaces ${ }^{19,27,28}$ (for example, $\mathrm{Au} / \mathrm{MoS}_{2}, \mathrm{BN} / \mathrm{graphene}$ and $\mathrm{WSe}_{2} / \mathrm{Bi}_{2} \mathrm{Se}_{3}$ ) is also around $0.2 \mathrm{~nm}$.

\section{vdW devices before and beyond two dimensions}

Early examples of vdW integration date back to the early 2000s, when various synthetic nanostructures were integrated into functional devices using simple physical-assembly approaches (such as drop-casting, rubbing and stamping). This enabled the early blossoming of nanoscale electronic and optoelectronic devices assembled from carbon nanotubes ${ }^{29,30}$ or semiconducting nanowires ${ }^{31,32}$. These pioneering efforts have shown the unique opportunities of vdW integration to 


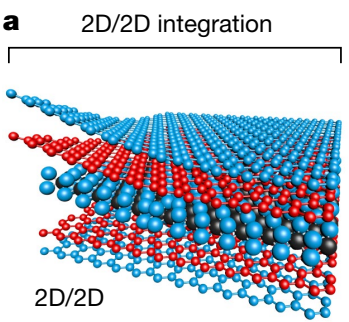

c

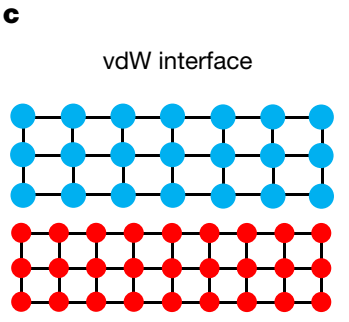

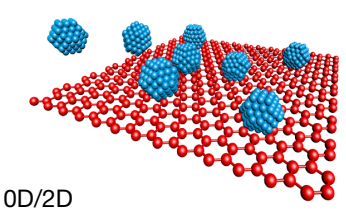

d

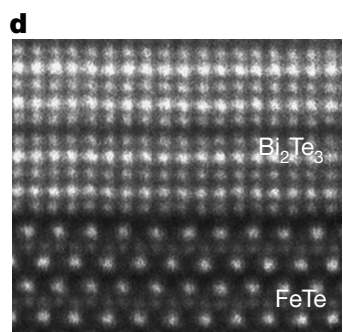

vdW integration beyond 2D materials

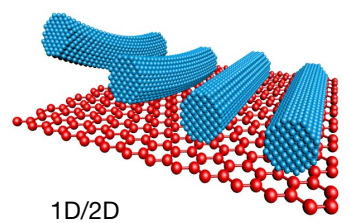

e

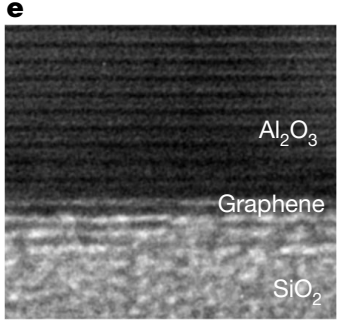

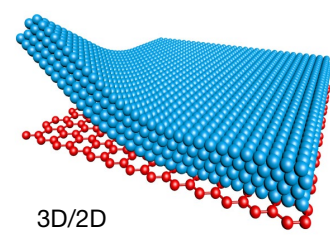

f

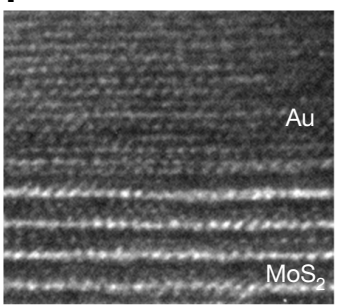

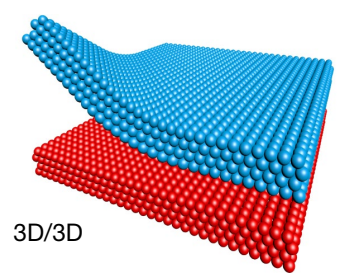

g

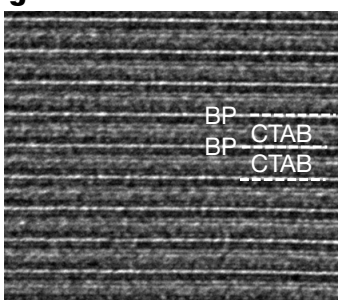

Fig. 2 Illustration and structural characteristics of vdW-integrated interfaces. a, b, Schematic illustrations of $2 \mathrm{D} / 2 \mathrm{D}$ integration (a) and $\mathrm{vdW}$ integration beyond $2 \mathrm{D}$ materials $(\mathbf{b})$. Blue and red spheres represent atoms of the integrated and the host material, respectively. c, Bondingfree atomic structure at a vdW interface. $\mathbf{d}-\mathbf{g}$, Cross-sectional images of vdW-contacting interfaces with various material dimensions: $\mathrm{Bi}_{2} \mathrm{Se}_{3} / \mathrm{FeTe}$ (d; adapted from ref. ${ }^{15}$, Springer Nature); $\mathrm{Al}_{2} \mathrm{O}_{3} /$ graphene (e; adapted

overcome the limitations of conventional epitaxial approaches to seamlessly combine highly disparate materials that are otherwise incompatible (for example, GaN, CdS or CdSe on $\mathrm{Si})^{32-34}$ and thus enable the creation of vdW devices with unprecedented functions and performance.

With the isolation of graphene ${ }^{35}$ and diverse $2 \mathrm{D}$ atomic crystals ${ }^{36}$ with dangling-bond free surfaces, vdW integration has gained tremendous momentum for creating a wide range of heterostructures with atomically clean and electronically sharp interfaces, offering a rich playground for both fundamental studies as well as novel device concepts $^{14,16,17,37}$ (Fig. 4). In fact, one of the primary engines for the from ref. ${ }^{18}$ with permission from PNAS); $\mathrm{Au} / \mathrm{MoS}_{2}$ (f; adapted from ref. ${ }^{19}$, Springer Nature); a high-order BP/CTAB superlattice (BP, black phosphorus; CTAB, cetyl-trimethylammonium bromide) (g; adapted from ref. ${ }^{20}$, Springer Nature). These weakly interacting vdW interfaces feature atomically clean and electronically sharp interfaces without apparent strain or disorder beyond the interface.

recent blossoming of $2 \mathrm{D}$ device research is the vdW integration itself, which makes the highest-quality heterostructures readily available to physicists and electrical engineers, without the need of the expensive facilities and lengthy development processes that are necessary for high-quality heterostructure growth.

$2 \mathrm{D} / 2 \mathrm{D}$ vdW heterostructures with an atomically clean, electronically sharp interface offer a rich system for fundamental studies and electronic device demonstrations ${ }^{27,38-53}$. For example, by encapsulating graphene in a $\mathrm{BN} /$ graphene/BN vdW heterostructure, an ultrahigh carrier mobility of $140,000 \mathrm{~cm}^{2} \mathrm{~V}^{-1} \mathrm{~s}^{-1}$ and up to $1,000,000 \mathrm{~cm}^{2} \mathrm{~V}^{-1} \mathrm{~s}^{-1}$ can be achieved at room temperature and cryogenic temperature,

a

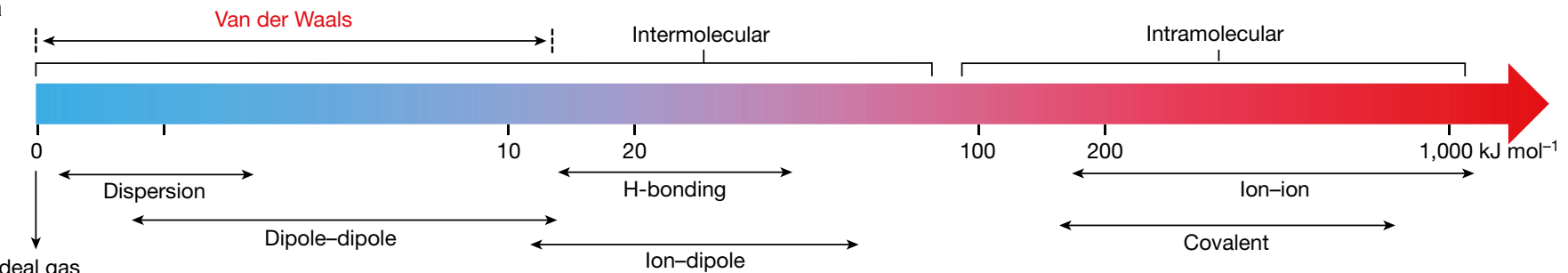

Ideal gas

b

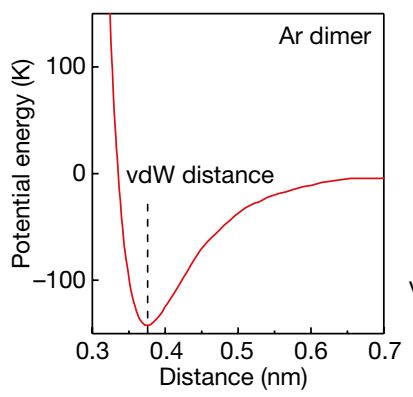

c

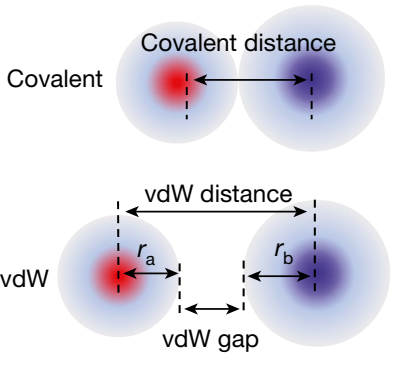

Fig. 3 Definition of vdW interaction, vdW distance and vdW gap. a, Energies of various molecular interactions. vdW interaction is the weakest intermolecular interaction (also termed physical interaction), much smaller than typical intramolecular interactions (also termed chemical interactions). b, Potential energy versus distance for an Ar dimer system, with a vdW distance of about $0.38 \mathrm{~nm}$ (lowest potential point) and a vdW gap of about $0.23 \mathrm{~nm}$ (difference between vdW distance and

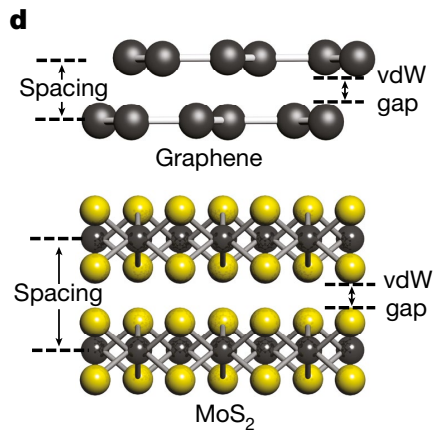

e

\begin{tabular}{lll}
\hline Material & Spacing & vdW gap \\
\hline Ar dimer & & $0.23 \mathrm{~nm}$ \\
BN & $0.33 \mathrm{~nm}$ & $0.19 \mathrm{~nm}$ \\
Graphene & $0.34 \mathrm{~nm}$ & $0.20 \mathrm{~nm}$ \\
Phosphene & $0.53 \mathrm{~nm}$ & $0.17 \mathrm{~nm}$ \\
MoS $_{2}$ & $0.62 \mathrm{~nm}$ & $0.18 \mathrm{~nm}$ \\
InSe & $0.84 \mathrm{~nm}$ & $0.17 \mathrm{~nm}$ \\
\hline
\end{tabular}

covalent radius). Image adapted from ref. ${ }^{24}$ with the permission of AIP Publishing. c, Schematic illustration of the vdW gap and the vdW distance in a covalent-bonded system and a vdW system. d, e, Comparison between layer spacing and calculated $\mathrm{vdW}$ gap in various layered $2 \mathrm{D}$ materials. The calculated vdW gap is around $0.2 \mathrm{~nm}$, comparable to that of the Ar dimer. 


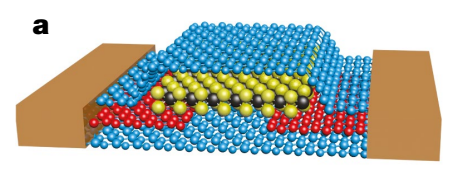

2D/2D FET

$$
\text { e }
$$

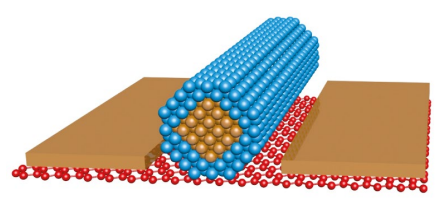

1D/2D high-speed transistor

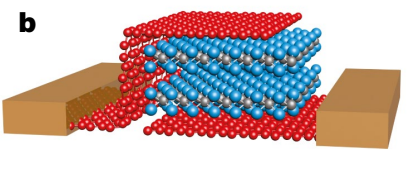

2D/2D GMR

$\mathbf{f}$

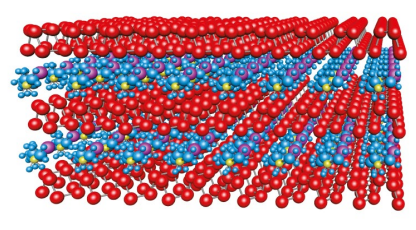

2D/OD superlattice

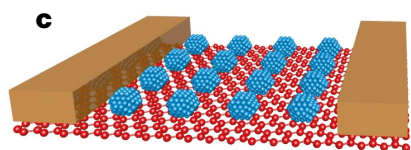

OD/2D photodiode

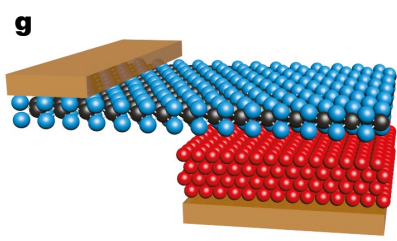

2D/3D TFET

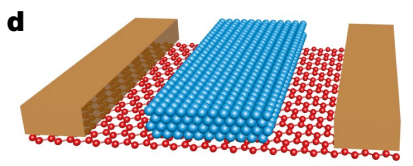

$1.5 \mathrm{D} / 2 \mathrm{D} \mathrm{FET}$

h

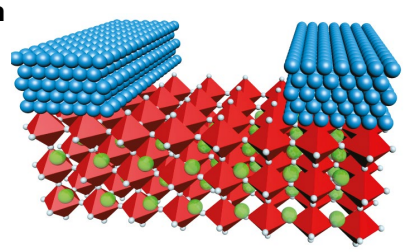

3D/3D photoconductor

Fig. 4 | Schematics of state-of-the-art vdW-integrated electronic and optoelectronic devices. a, 2D/2D planar transistor with a $2 \mathrm{D}$ semiconductor as the channel ( $\mathrm{MoS}_{2}$, yellow and black), a 2D dielectric (BN, blue) as the encapsulation layer and a 2D metallic material (graphene, red) as the contact electrodes. b, 2D/2D vdW magnetic vertical tunnelling junctions, with atomically thin $\mathrm{CrI}_{3}$ (blue) as a spin-filter tunnel barrier and graphene (red colour) as the contact electrodes. GMR, giant magnetoresistance. c, $0 \mathrm{D} / 2 \mathrm{D}$ photodiode created by vdW integration of quantum dots or plasmonic nanoparticles (blue) on top of graphene (red) without damaging the pristine graphene lattice, thus providing greatly enhanced photocurrent. d, $1.5 \mathrm{D} / 2 \mathrm{D}$ top-gate FET based on the vdW integration of a $1.5 \mathrm{D} \mathrm{Al}_{2} \mathrm{O}_{3}$ nanoribbon (as the dielectric; blue) on top of graphene (red) without damage in its pristine lattice. e, $1 \mathrm{D} / 2 \mathrm{D}$ highspeed transistor obtained by vdW-integrating a $1 \mathrm{D}$ core-shell nanowire

respectively $13,27,54$. Similar vdW structures can be created by vdWintegrating a $2 \mathrm{D}$ semiconducting channel, a 2D insulating dielectric and 2D metallic contacts (Fig. 4a), enabling the realization of quantum oscillation and the highest carrier mobility in various $2 \mathrm{D}$ semiconductors $^{55-59}$. Besides conventional planar structures, another key development in $2 \mathrm{D} v \mathrm{vdW}$ devices is the demonstration of vertical transistors by sandwiching a $2 \mathrm{D}$ insulator or semiconductor channel between graphene electrodes, enabling a switchable vertical transport mechanism based on a tunable tunnelling or thermionic barrier ${ }^{39,41,42,44,60}$. Similarly, vertical graphene/ $\mathrm{MoS}_{2}$ junctions have enabled the construction of unique gate-tunable photodiodes ${ }^{42,44,46}$, and a $\mathrm{MoS}_{2} / \mathrm{BN}$ quantum well structure has been used to create LEDs ${ }^{49}$.

Besides high-performance devices, vdW heterostructures have also provided an ideal platform for fundamental studies. For example, vdW-assembled hetero-bilayers host electrically tunable interlayer excitons with electrons and holes localized in different layers ${ }^{61-64}$, inheriting novel valley-contrasting physics from their monolayers but with much longer exciton lifetimes. vdW heterostructures of $2 \mathrm{D}$ magnetic materials have allowed probing intrinsic 2D magnetism (for example, $\mathrm{CrI}_{3}$ ) and realizing giant tunnelling magnetoresistance ${ }^{65-70}$ endowed by the multiple spin-filtering effect (Fig. 4b). In addition, the discovery of 2D magnetic materials could enable the creation of $\mathrm{vdW}$ magnetic heterostructures through the proximity effect ${ }^{71}$, which is expected to be strong in 2D materials, for exploring a variety of novel phenomena and functionalities. Beyond the chemical composition of the constituent layers, the twist angles between different layers offer another degree of freedom to tailor their electronic properties for exotic physics, as highlighted by the recent observation of correlated insulator behaviour and superconductivity in magic-angled bilayer graphene homostructures ${ }^{72,73}$.

Beyond 2D materials, the vdW integration of $2 \mathrm{D}$ atomic layers with other dimensional material components, such as $0 \mathrm{D}, 1 \mathrm{D}$ or $3 \mathrm{D}$ bulk materials, is essential for integrating $2 \mathrm{D}$ materials into functional devices; the related integrating approaches are summarized in Box 1 . The previous PVD integration can usually damage underlying monolayer atomic lattices and degrade their electronic properties ${ }^{74}$; hence, the development of a damage-free vdW integration approach is essential for capturing the intrinsic merits of $2 \mathrm{D}$ materials in functional

(as a self-aligned mask, blue) to enable the construction of $2 \mathrm{D}$ transistors (graphene, red) with high cut-off frequency. f, 2D/0D high-order superlattice obtained by intercalating $0 \mathrm{D}$ molecules (blue) into a $2 \mathrm{D}$ material (phosphorene, red), resulting in a stable superlattice with radically different constituents and tunable interlayer distances or band offset. g, 2D/3D tunnelling transistor constructed using 2D $\mathrm{MoS}_{2}$ (blue and black) and 3D Ge (red). The vdW integration of $\mathrm{MoS}_{2}$ enables the creation of an electronically abrupt junction for high-efficiency electron tunnelling, and 3D germanium provides well controlled doping density and the desired electron affinity for ultra-small subthreshold swing. h, 3D/3D vdW integration enables the creation of damage-free metal contacts (blue) on delicate perovskite (red), with much more efficient charge transport than that achieved with deposited contacts.

devices. For example, damage-free vdW integration of 0D plasmonic nanostructures ${ }^{75}(\mathrm{Au})$ or quantum $\operatorname{dots}^{76}(\mathrm{PbS})$ on graphene has enabled the fabrication of graphene photodetectors with greatly enhanced photocurrent (Fig. 4c). vdW integration of 1.5-dimensional (referred to as nanoribbon) $\mathrm{Al}_{2} \mathrm{O}_{3}$ on graphene opens a bond- and damage-free dielectric integration pathway to high-mobility top-gated graphene transistors and predates $2 \mathrm{D} / 2 \mathrm{D}$ vdW integration (Fig. $4 \mathrm{~d})^{18}$. Similar approaches were also used to integrate nanowire gates with graphene nanoribbons to ensure high performance in graphene nanoribbon transistors ${ }^{77,78}$. Furthermore, vdW-integrating a metal oxide core-shell nanowire or a $1 \mathrm{D}$ metal/oxide gate stack on $2 \mathrm{D}$ materials defines a self-aligned ultrashort channel length without damaging the underlying 2D semiconductors (Fig. 4e), enabling the realization of the fastest so far transistors from graphene and $\mathrm{MoS}_{2}$, with an intrinsic cut-off frequency of $420 \mathrm{GHz}$ and $42 \mathrm{GHz}$, respectively ${ }^{79-82}$. In particular, the physical assembly of lithographically defined gate arrays on large-area $2 \mathrm{D}$ atomic layers opens a pathway to scalable vdW integration ${ }^{81,82}$. Beyond low-dimensional nanostructures, molecules or ions can be chemically absorbed onto 2D materials to form self-assembled monolayers (SAM) and 2D/SAM vdW heterostructures ${ }^{83,84}$, or intercalated into layered crystals or $2 \mathrm{D} / 2 \mathrm{D}$ vdW heterostructures ${ }^{20,85}$, generating high-order bond-free vdW superlattices between radically different constituents with highly tunable interlayer distances and tailored electronic properties (Figs. 2h, $4 \mathrm{f}$ ).

vdW-integrated devices can also be extended to 3D materials. This approach combines the atomically sharp interfaces provided by vdW integration with well developed 3D systems with rich material choices and designable properties. One interesting example is the creation of a tunnelling transistor with ultralow sub-threshold swing from a $2 \mathrm{D} / 3 \mathrm{D}$

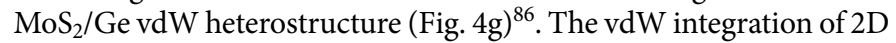
$\mathrm{MoS}_{2}$ enables the creation of an electronically abrupt junction, which is critical for efficient electron tunnelling (not easily achievable within ion-implanted heterostructures), whereas the use of 3D germanium provides a well controlled doping density and the desired electron affinity (difficult to achieve using $2 \mathrm{D}$ semiconductors) to maximize the ON-state current while retaining low OFF-state current and small sub-threshold swing. Another recent example is the demonstration of vdW-integrated metal/semiconductor junctions (3D/2D) with highly 
Box I

\section{Building blocks for vdW integration}

A variety of materials can function as the building blocks for vdW integration (Figure). Although integration methods may vary, they all share similar integration processes, with three essential steps: (1) pre-fabrication of the vdW building blocks, (2) isolation from the sacrificial substrate and (3) physical assembly (or lamination) on the target material.

\section{Molecules, OD and 1D materials}

Molecules and OD and 1D materials are generally produced by bottom-up chemical synthesis as freestanding objects in solution or with low bonding forces to a growth substrate ${ }^{111}$. They can function as vdW building blocks and be directly integrated on the target material using simple surface adsorption, dip-coating, drop-casting or physical rubbing processes, as shown in Figure. Scalable integration may also be achieved by assembling these nanostructures (using fluid ${ }^{112}$, electrical ${ }^{113}$ or magnetic-field-guided ${ }^{114}$ assembly) directly on the target material, or by first creating them on a sacrificial substrate using advanced lithography and then transferring them onto the target material ('assemble-peeling' in Figure) ) $^{75,81}$.

\section{D materials}

Most 2D layered materials (for example, graphite and $\mathrm{MoS}_{2}$ ) can be directly integrated on the target material using top-down methods, such as rubbing or mechanically exfoliation ${ }^{115}$. On the other hand, 2D building blocks and more complicated heterostructures can be synthesized using various bottom-up approaches (such as CVD) on a sacrificial substrate, then isolated using mechanical peeling (dry transfer) ${ }^{116}$, solution-assisted peeling ${ }^{117,118}$, metal-assisted transfer ${ }^{119-123}$ or an etching process (wet transfer) ${ }^{124}$, and then laminated onto the target material. Large-scale $2 \mathrm{D}$ $\mathrm{vdW}$ thin films are also achieved through solution assembly of colloidal 2D nanosheets, featuring broad-area dangling-bond-free 2D/2D contacts (between nanosheets) with few interfacial trapping states ${ }^{125,126}$

\section{D materials}

The key challenge for vdW integration of 3D materials is their rigidity and relatively large surface roughness, leading to a partially contacting interface, which is undesirable for stable vdW integration and charge transport. 3D metals pre-deposited using PVD or 3D polymers prefabricated using solution coating typically demonstrate weak bonding with the substrate and can be mechanically isolated ${ }^{19}$ using a 'peeling' method similar to that used for 2D building blocks. The back surface of these 3D materials replicates that of the sacrificial substrate and can be atomically flat for effective vdW integration. However, the top surface of PVD metals typically exhibits nanometre-scale roughness ${ }^{127}$ and may not be optimal for vdW integration ${ }^{128,129}$.

For 3D semiconductors pre-fabricated using chemical approaches (such as MOCVD and MBE) with strong interface bonds, selective etching of the sacrificial layer is necessary for their isolation. For example, single-crystal silicon with a smooth surface can be isolated from a siliconon-insulator structure ${ }^{105}$. For high-quality III-V semiconductors, a pre-patterning process (into stripes; 'etch-peeling' in Figure) can help reduce the etching time and provide improved surface flatness and mechanical flexibility; the isolation and integration of InAs (from AISb), GaAs (from AIAs) and GaN (from AIN) have been demonstrated ${ }^{93,97,99}$. Additional functional or passivation layers may also be deposited on the isolated building blocks before vdW integration for further tailoring of the interface properties.

Methods
$\begin{gathered}\text { Casting } \\ \text { (OD, 1D, 2D) }\end{gathered}$
$\begin{gathered}\text { Rubbing } \\ \text { (OD, 1D) }\end{gathered}$
$\begin{gathered}\text { Taping } \\ \text { (2D) }\end{gathered}$
Assemble-peeling
(OD, 1D, 2D)
Peeling
(CVD 2D, PVD 3D
metal)

Box Fig. $1 \mid$ 0D, 1D, 2D and 3D building blocks for vdW integration. Blue colour represents the integrated material, red colour represents the host material, dark grey and light grey represent the substrate material and sacrificial layer, respectively. The host material is involved in the isolation process for direct integration, but not involved in the isolation process of indirect integration. Details are provided in Box 1. 
tunable barrier height approaching the Schottky-Mott limit ${ }^{19}$. The use of a dangling-bond-free 2D semiconductor is essential for avoiding Schottky-Taam surface states (not easily achievable in covalent-bonded 3D semiconductors), and the use of a 3D metal provides a rich material library with proper work functions for the designed band alignments (not yet available in 2D metals). In optoelectronics, various devices are also demonstrated by vdW-integrating $2 \mathrm{D}$ graphene with a $3 \mathrm{D} \mathrm{Si}$ waveguide, a $\mathrm{SiN}_{x}$ ring resonator or optical fibre, allowing the realization of a unique gate-tunable optical modulator ${ }^{87}$, frequency combs ${ }^{88}$ and a Q-switched laser ${ }^{89,90}$, respectively. Besides electron transport and electron-photon interaction, the recently emerged strong electronphonon coupling in a 2D/3D vdW structure could also offer a rich system for exploring new physics and devices, such as strong Raman scattering $\left(\mathrm{WSe}_{2} / \mathrm{SiO}_{2}\right)^{91}$ and high-critical-temperature superconductivity $\left(\mathrm{FeSe} / \mathrm{SrTiO}_{3}\right)^{92}$.

$3 \mathrm{D} / 3 \mathrm{D}$ vdW heterostructures may also be created with unique attributes $^{93-95}$. For example, $3 \mathrm{D}$ metal thin films can be vdW-integrated onto $3 \mathrm{D}$ semiconductors as contact electrodes with minimum interfacial disorder, greatly reducing the interface states and pinning effects in typical metal/semiconductor junctions and allowing the generation of a highly tunable Schottky barrier largely dictated by the metal work function ${ }^{19}$. This low-energy metal integration is crucial for forming $3 \mathrm{D} / 3 \mathrm{D} \mathrm{vdW}$ metal/semiconductor contacts from delicate materials, such as organic polymer ${ }^{96}$ or hybrid perovskites (Fig. $4 \mathrm{~h}$ ), where the contact area could be severely damaged by the conventional high-energy metal deposition process. Our preliminary studies show that a vdW metal/perovskite contact features a damage-free and atomically clean interface, allowing much more efficient charge transport and achieving a highest optical gain of over $10^{9}$. Additionally, distinct 3D semiconductor membranes may be separated from their epitaxial growth substrates ${ }^{97-99}$ and physically assembled to enable the creation of new-generation $3 \mathrm{D} / 3 \mathrm{D}$ semiconductor heterostructures or superlattices beyond the limits of traditional material-growth approaches.

\section{Integration by design}

The initial studies of vdW integration and vdW heterostructures revealed many exciting opportunities. By further extending vdW integration to include the well developed 3D materials and other low-dimensional materials, a much broader range of material components can be isolated, mixed, matched and combined to create highly engineered heterostructures and enable the construction of a new class of electronic, optoelectronic or magnetic devices with unprecedented performance or entirely new functions beyond the reach of the existing materials. Here we discuss some unique opportunities arising from vdW-integrated heterostructures.

\section{Tunable vdW metal/semiconductor junctions}

In vdW metal-semiconductor (MS) junctions, a metal thin film is pre-fabricated and physically laminated onto the semiconductor surface with little integration-induced damage and minimized FLP effect, thus ensuring a highly tunable barrier height by design ${ }^{19}$. Therefore, Ohmic contacts to various $2 \mathrm{D}$ semiconductors may be realized by $\mathrm{vdW}$-integrating specific metals with proper work functions matching the respective semiconductor band edges. In this way, the majority-carrier type can be tailored from electrons to holes by tuning the work function of the contact metals, and complementary metal-oxide-semiconductor (CMOS) circuits could be created with the same 2D channel material by simply integrating metals with work functions matching the conduction- or valence-band position. We note that CMOS integration using a single $2 \mathrm{D}$ semiconductor is a key challenge for the $2 \mathrm{D}$ device community because there is little physical space for impurity dopants in such atomically thin semiconductors.

vdW metal integration may also be explored for improving the contacts to various 3D semiconductors; however, the effect may not be as straightforward as in 2D semiconductors. Typical 3D semiconductors could suffer from surface dangling bonds owing to the crystal lattice termination at the surface, leading to unavoidable interface states.
Specifically, for a covalent semiconductor (such as Si and GaAs) with little electronegativity difference, dangling bonds result in a large number of gap states that serve as reservoirs for carriers to pin the Fermi level. In this case, vdW integration may achieve limited improvement, but could still be observable once the surface dangling bonds are passivated (for example, H-termination or thin oxide passivation) ${ }^{40,100}$. On the other hand, for covalent semiconductors with large electronegativity difference (for example, $\mathrm{SiC}$ or $\mathrm{GaN}$ ) or ionic semiconductors, a minimized FLP effect with improved contact behaviour is expected. This could be particularly useful for large-bandgap III-V semiconductors (for example, GaN), where the optimized p-type contact is difficult to achieve using a conventional doping approach.

Besides enhancing carrier transport efficiency, vdW-integrated MS junctions could also benefit devices requiring a high Schottky barrier to hinder charge transport. For example, in metal-semiconductor field-effect transistors (MESFETs), the MS Schottky barrier is used as the gate to modulate carrier concentration inside the channel ${ }^{1}$. In this case, a large Schottky barrier is desired for the gate to ensure smaller gate leakage and larger gate voltage operation range, but is hard to control using a conventional deposited MS junction owing to the FLP effect. With the ability to create vdW MS junctions with tunable barrier height, optimized Ohmic contacts and Schottky gate could be achieved at the same time by integrating metals with work functions matching either the band edges (small barrier for contacts) or the middle of the forbidden gap (large barrier for the gate) (Fig. 5a). Furthermore, designable Schottky barriers at the vdW MS interfaces could also enable the construction of novel devices, such as hot-electron photodetectors with tunable and designable detection edge or multi-level memory cells.

\section{Pinning-free interfaces for coherent transport}

Coherent charge injection is critical for various quantum electronic devices that require phase coherence, but is often plagued by interface scattering due to structural disorder at the electrode interfaces. For example, in magnetoresistance and spintronic devices based on polarized carrier transport across various heterostructure interfaces, such as ferromagnetic/conductor/ferromagnetic (giant magnetoresistance), ferromagnetic/insulator/ferromagnetic (tunnel magnetoresistance) or ferromagnetic/semiconductor/ferromagnetic (spin transistor), the spin-injection efficiency is dictated by the interface quality and largely suppressed by interfacial roughness, impurities and other types of interface disorder. For instance, in a deposited Fe/Si magnetic junction, high-energy Fe atoms (during vacuum deposition) tend to diffuse into the Si lattice and lead to randomly oriented local magnetic moments ${ }^{101}$, severely scattering the injected spin-polarized electrons and degrading overall spin polarization. By contrast, within vdW magnetic junctions, the interface states and the atom interdiffusion-induced scattering effects could be minimized by physically integrating various functional components (ferromagnetic metal, insulator, semiconductor) with atomically clean and magnetically sharp interfaces, enabling efficient coherent spin injection ${ }^{96}$ (Fig. 5b). Beyond magnetic junctions, the vdW integration approach may also be extended to create other functional junctions that were previously plagued by interface disorder. Possible directions include vdW superconductor/semiconductor junctions, Josephson tunnelling junctions (Fig. 5c) and metal/insulator tunnelling junctions.

\section{Heterogeneous-layer assembly by design}

One of the most attracting attributes of vdW integration is the freedom of 'assembly-by-design'. A possible playground for vdW integration, therefore, would be stacks of III-V semiconductor-based devices that could not be previously grown (through MBE or MOCVD). For instance, a heterojunction bipolar transistor based on the wide-bandgap $\mathrm{GaN}$ is commercially important for high-frequency, high-power amplification $^{102}$, but its overall performance is severely limited by the low conductivity of MOCVD-grown p-type GaN base region ${ }^{103}$. Using vdW layer-by-layer integration, one could take a 'transplant surgery' 


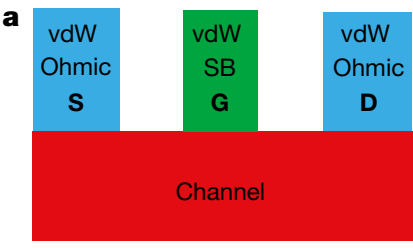

Tunable energy barrier

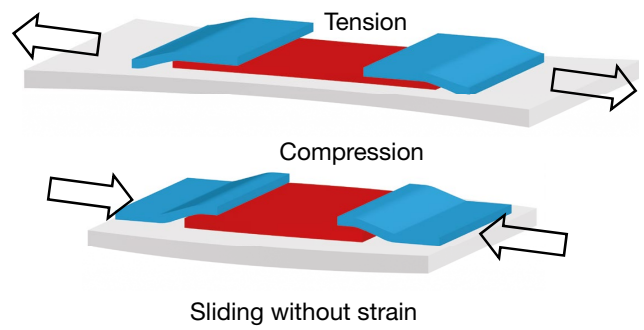

Fig. 5 | New opportunities in vdW-integrated heterostructures and superlattices beyond 2D materials. a, A MESFET structure using onestep vdW integration of pre-fabricated metal electrodes with distinct work functions, resulting in a low Schottky barrier (SB) (source, S, and drain, D) and a large Schottky barrier (gate, G) through a single integration step. $\mathbf{b}, \mathbf{c}$, Schematic illustration of a vdW-integrated ferromagnetic (FM)/semiconductor junction (b) and a superconductor/ insulator/superconductor junction (c) for coherent charge injection in quantum electronic devices. The defect- and pinning-free interface could considerably reduce interface disorder and the associated scattering that plague such devices. d, Heterostructure integration of $n-G a N / p-W e_{2} /$ $\mathrm{n}$-GaN vdW structure (B, base; C, collector; E, emitter) for high

approach to replace the poor p-type GaN layer with other materials of better p-type conductivity (for example, p-type $\mathrm{WSe}_{2}$ or p-type $\mathrm{Si}$ ),

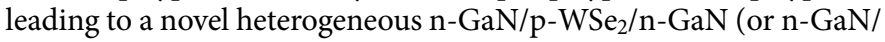
p-Si/n-GaN) structure (Fig. 5d). Similarly, vdW stacking could also play an important role in organic polymer stacks that typically share similar material solubilities ${ }^{104}$ and are hard to be integrated without dissolving others, or amorphous oxide stacks that share similar etchants and are limited by the lack of selective etching process. Possible applications include vdW vertical memories, vdW tandem photovoltaics, vdW OLEDs (organic LEDs), bipolar OFETs (organic field-effect transistors) and organic CMOS circuits.

\section{vdW sliding interface}

Flexibility and stretchability are important features for future wearable electronic devices, but are difficult to achieve in typical silicon or III-V semiconductor wafers. Previous approaches to this challenge include two conceptually different methods. One relies on the use of new structures (such as wavy silicon membranes) ${ }^{105}$ and the other relies on new materials that are intrinsically flexible (such as organic polymers). Although these approaches have successfully improved the flexibility of individual building blocks, interface strain in heterostructures, which could dictate the overall device flexibility, has been insufficiently addressed until now. When a chemically bonded heterostructure is stretched, large internal strains are usually built up at the interface between two dissimilar materials (for example, semiconductor/dielectric, semiconductor/metal), which typically have distinct Young's moduli, where the failure often occurs first. By contrast, within vdW-integrated devices, constituent components with distinct mechanical properties could slide over each other when compressed or stretched (Fig. 5e) while retaining clean and sharp vdW interfaces with optimized charge transport. Such a sliding junction can effectively release the local strain at the interface and therefore overcome the fundamental mechanical mismatch in functional systems. Other sliding-related effects, such as the triboelectric effect or frictional heat generation, are also interesting to investigate.
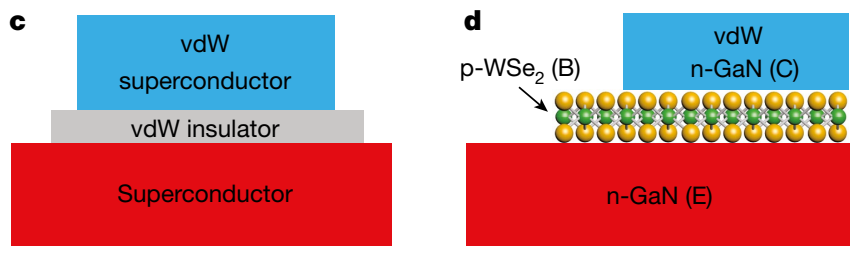

Assembly by design

g

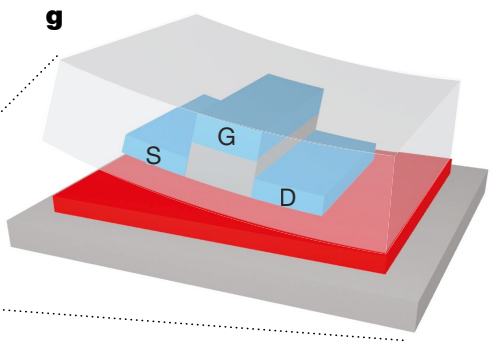

Plug and probe

performance heterojunction bipolar transistor by replacing $\mathrm{p}-\mathrm{GaN}$ with p-type WSe $\mathrm{W}_{2}$. e, Schematic illustration of a vdW heterostructure (vdW metal, blue; stretchable semiconductor, red) with distinct stiffness under mechanical compression (bottom) and tension (top). The bonding-free vdW interaction allows two contacting materials to slide against each other and effectively release the local strain at the interface. f, g, Plugand-probe vdW integration, where a complex structure (for example, source drain contacts and a gate stack) is pre-fabricated on wafer scales. Every time used, one piece of the structure is cut from the mother wafer and laminated on the target material within a few seconds for directly probing its intrinsic properties.

\section{Plug-and-probe intrinsic material properties}

vdW integration could also be extended to integrate multiple functional components through a 'one-step' assembly process without additional lithography. For example, all essential components of a typical MOSFET (gate metal, dielectric insulator, contact metal and encapsulation polymer, as shown in Fig. 5f, g) could be pre-fabricated in a large scale and directly laminated on the targeted semiconductor surface. Besides enabling optimized contact and dielectric interfaces, such vdW integration offers an effective 'plug-and-probe' approach for investigating intrinsic material properties. The top gate and contact structures could be integrated on the target material without any conventional fabrication steps or exposure to solvents and, more importantly, the entire pre-fabricated structures could be integrated within a few seconds at room temperature, which would be crucial for many unstable materials (such as silicene or germanene ${ }^{106,107}$ ) that rapidly degrade with time or fabrication steps. Besides these exotic $2 \mathrm{D}$ materials, the relatively 'old' 2D materials (such as graphene and transition metal dichalcogenides) also deserve a re-visit through this simple plug-andprobe approach. Our preliminary results demonstrate that the effective field-effect mobility of CVD-grown monolayer $\mathrm{WSe}_{2}$ could be substantially improved (up to about $200 \mathrm{~cm}^{2} \mathrm{~V}^{-1} \mathrm{~s}^{-1}$ ) by an immediate plug-and-probe operation, suggesting that the performance of other $2 \mathrm{D}$ materials may be largely underestimated owing to unnoticed time-related or fabrication-induced degradations.

Beyond 2D materials, this low-temperature, solvent-free and low-energy integration strategy could be useful for other delicate materials, including molecular monolayers, organic thin films or crystals, and metal halide perovskites. Such materials are usually not stable under high temperature, not compatible with traditional micro-fabrication processes (for example, they are soluble in various solvents) or are highly prone to metal-deposition-induced degradation. Importantly, the plug-and-probe approach offers a lithography-free, solvent-free and damage-free pathway to rapid device prototyping using these delicate materials, which is essential for investigating their intrinsic properties and pushing the performance limit of various devices (OFETs, OLEDs, 

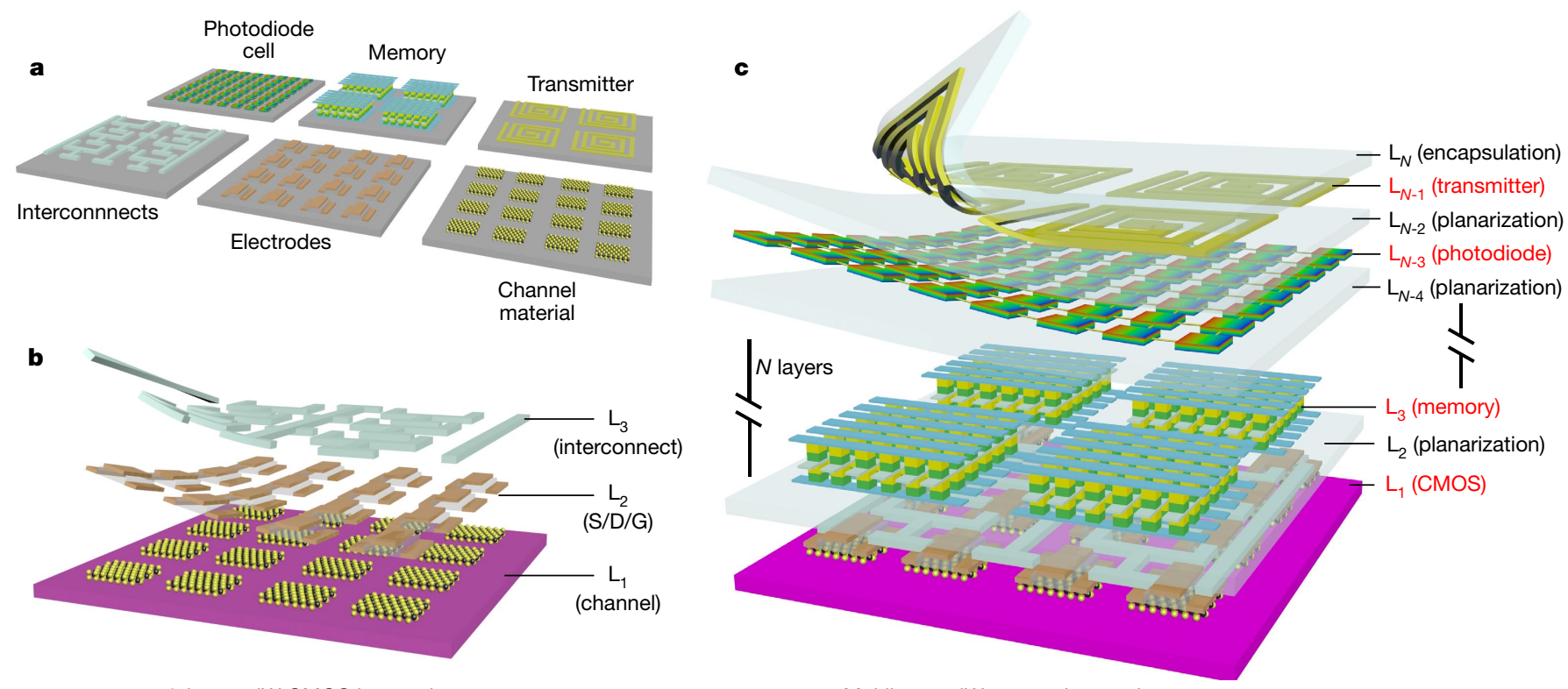

3-layer vdW CMOS integration

Multilayer vdW system integration

Fig. 6 | Potential layer-by-layer vdW assembly of a 3D electronic system. a, Schematics of the essential building blocks for vdW device integration and the essential device layers for vdW system integration. b. Schematic illustration of circuit-level (CMOS circuit) vdW integration by assembling various building blocks, including channel material $\left(\mathrm{L}_{1}\right)$, electrodes

organic photovoltaics and perovskite FETs, photovoltaics and LEDs) previously plagued by fabrication-induced degradations.

Another advantage of the plug-and-probe approach is its energy and cost efficiency. In conventional micro- and nano-electronics research, device fabrication involves time-consuming, expensive lithography and vacuum deposition processes that may not be easily accessible in many laboratories. Furthermore, precious metals (for example, $\mathrm{Au}$ and $\mathrm{Pt}$ ) have to be deposited uniformly across a metre-scale vacuum chamber, even when only a few micrometre-scale devices are fabricated each time. In contrast, using the plug-and-probe approach, a large array of standard device structures could be batch-processed on the wafer scale using conventional lithography with high throughput. When needed, a small area of the device structure is isolated from the mother wafer (Fig. 5f, g) and laminated onto the target material like a 'tattoo tape', with greatly reduced time and cost. This is especially desired for structures involving more sophisticated processes, such as extreme ultraviolet photolithography-processed ultrashort gates, ultrathin dielectrics or special structures (for example, optical metamaterials and T-gates). We therefore anticipate that these complex pre-fabricated wafers could be the first products using plug-and-probe commercialization and standardization, which may further lower the entry barrier for the broader research community, greatly accelerate device prototyping from new materials and create new opportunities in nanoelectronics and nanophotonics.

\section{Outlook}

After nearly two decades of expanding efforts in vdW integration of various material systems, questions are frequently raised on whether this is purely an academic game or it could offer some practical technological potential. The recent demonstrations of $\mathrm{vdW}$ integration of large arrays of lithographically defined microstructures of 3D materials ${ }^{79,81,82}$ could alter the landscape and offer a promising outlet by combining the advantages of vdW integration (for example, atomically clean and electronically sharp interfaces) with more scalable and reliable $3 \mathrm{D}$ materials and conventional fabrication techniques. For example, the vdW-integrated 3D metal/MoS $\mathrm{M}_{2}$ interface has enabled the creation of a nearly ideal pinning-free MS interface, which is difficult to achieve with conventional fabrication approaches ${ }^{19}$, allowing the experimental validation of the Schottky-Mott limit predicted many and dielectrics $\left(\mathrm{L}_{2}\right)$ and interconnects $\left(\mathrm{L}_{3}\right)$ on a target semiconductor layer. c, Schematic illustration of high-order system-level integration by vdW-stacking multiple active functional layers with low-temperature planarization layers (passive layers; translucent in the schematic) in between.

decades ago. The creation of such high-performance devices with simple vdW integration highlights the robustness of this approach and its unique merits for creating pristine interfaces that are not otherwise readily accessible.

vdW integration can be further extended for scalable system-level integration. Various active or passive device layers can be assembled by repeatedly laminating pre-fabricated vdW building blocks (for example, semiconductors, gate dielectrics and contacts) or pre-assembled device layers (such as CMOS circuits, flash memories and photodiode cells) using a layer-by-layer stacking process (Fig. 6). Each active device layer could be separated by a passive planarization layer and may be further connected with other layers using vertical interconnect access holes. Within this heterogeneous stacking geometry, function layers are vdW integrated without the limits set by lattice-matching or process-compatibility requirements, which could enable $3 \mathrm{D}$ electronic integration with much reduced process cost and device footprint. Hence, such a combination of vdW integration with more mature and more reliable 3D materials and devices could bridge the gaps between academic research and industrial applications.

Despite the extraordinary potential and many exciting demonstrations of proof-of-concept devices, the challenges of turning them into practical technologies should not be understated. Sustained investment is imperative, not only for pushing the limit of individual devices but also for improving their integration yield, processability, stability and scalability. Although the ability to physically assemble individual building blocks into vdW heterostructure has offered vast flexibility for heterogeneous material integration and has considerably accelerated both fundamental studies and proof-of-concept demonstrations, scalable $\mathrm{vdW}$ integration of heterostructure device arrays with high yield and throughput remains a major challenge. For example, the state-of-the-art vdW stacking process still largely relies on a manual alignment-transfer technique using a soft stamp (for example, silicone polymer), which typically has a large mechanical expansion coefficient and could limit the alignment resolution in large-scale integration. Additionally, for most vdW interfaces, the size, uniformity, wrinkles, surface contamination and interfacial air bubbles represent notable technical challenges that could severely limit the device yield, whereas these difficulties grow exponentially with increasing integration areas or integration steps ${ }^{108}$. These technical challenges require unified efforts from multiple 
disciplines, particularly chemistry and materials science, for the synthesis and fabrication of vdW building blocks with high uniformity, atomic flatness and elaborate structure design ${ }^{52,109,110}$, as well as engineering, for the development of an automatic stamping machine with appropriate mechanical design, which would be critical for reducing defects, improving the integration yield and achieving better alignment resolution over a large scale.

Another potential challenge is the reliability and stability of the weakly bonded vdW heterostructures. As noted, the vdW interactions between two fully contacting surfaces are strong enough to hold bulk materials together against gravitational movement or disintegration. Therefore, the instability or delamination of two fully vdW-interacting materials is unlikely, unless there is a strong enough external force or interfacial strain. On a related matter, thermal expansion and contraction of the individual vdW building blocks should not be overlooked, because mismatched thermal properties could lead to interfacial strain and interfacial sliding when the temperature changes. In this regard, the mild vdW integration process is advantageous because it normally does not require a large temperature swing, which is typically inevitable in conventional integration processes. However, such a thermal effect should still be considered during device operation, and strain-releasing structures or a heat-dissipation layer could be integrated to prevent accumulated strain in large-area integration. Despite these and other challenges, the bond-free vdW integration opens a new dimension for material integration, with unparalleled freedom to integrate materials that could not be previously combined, opening up new possibilities to approach physical limits previously inaccessible and to enable the development of novel devices with unprecedented performance or entirely new functions beyond the reach of existing materials and systems. Such exciting prospects and the associated new challenges call for more efforts to fully unlock the potential of $\mathrm{vdW}$ integration for future technologies.

Received: 5 August 2018; Accepted: 10 December 2018; Published online 20 March 2019.

1. Sze, S. M. \& Ng, K. K. Physics of Semiconductor Devices (John Wiley \& Sons, Hoboken, 2006).

2. Arthur, J. R. Molecular beam epitaxy. Surf. Sci. 500, 189-217 (2002).

3. Doering, R. \& Nishi, Y. Handbook of Semiconductor Manufacturing Technology (CRC Press, Boca Raton, 2007).

4. Kourkoutis, L. F., Song, J., Hwang, H. \& Muller, D. Microscopic origins for stabilizing room-temperature ferromagnetism in ultrathin manganite layers. Proc. Natl Acad. Sci. USA 107, 11682-11685 (2010).

5. Matthews, J. \& Blakeslee, A. Defects in epitaxial multilayers: I. Misfit dislocations. J. Cryst. Growth 27, 118-125 (1974).

6. Vallet, M. et al. Highly strained AlAs type interfaces in InAs/AISb heterostructures. Appl. Phys. Lett. 108, 211908 (2016).

7. Niehle, M., Rodriguez, J.-B., Cerutti, L., Tournié, E. \& Trampert, A. On the origin of threading dislocations during epitaxial growth of III-Sb on $\mathrm{Si}(001)$ : a comprehensive transmission electron tomography and microscopy study. Acta Mater. 143, 121-129 (2018).

8. Ni, Y. et al. Influence of the carbon-doping location on the material and electrical properties of a AlGaN/GaN heterostructure on Si substrate. Semicond. Sci. Technol. 30, 105037 (2015).

9. Gusev, E., Cabral, C. Jr, Copel, M., D'Emic, C. \& Gribelyuk, M. Ultrathin $\mathrm{HfO}_{2}$ films grown on silicon by atomic layer deposition for advanced gate dielectrics applications. Microelectron. Eng. 69, 145-151 (2003).

10. Wang, X., Tabakman, S. M. \& Dai, H. Atomic layer deposition of metal oxides on pristine and functionalized graphene. J. Am. Chem. Soc. 130, 8152-8153 (2008)

11. Chen, L. Solid state amorphization in metal/Si systems. Mater. Sci. Eng. Rep. 29, 115-152 (2000)

12. Tung, R. T. The physics and chemistry of the Schottky barrier height. Appl. Phys. Rev. 1, 011304 (2014).

13. Dean, C. R. et al. Boron nitride substrates for high-quality graphene electronics. Nat. Nanotechnol. 5, 722-726 (2010).

This paper reported the first 2D/2D vdW heterostructure between graphene and boron nitride with minimum interface disorder and examined the intrinsic electronic properties of graphene.

14. Geim, A. K. \& Grigorieva, I. V. Van der Waals heterostructures. Nature $\mathbf{4 9 9}$, 419-425 (2013).

15. He, Q. L. et al. Two-dimensional superconductivity at the interface of $a \mathrm{Bi}_{2} \mathrm{Te}_{3} /$ FeTe heterostructure. Nat. Commun. 5, 4247 (2014).

16. Novoselov, K., Mishchenko, A., Carvalho, A. \& Neto, A. C. 2D materials and van der Waals heterostructures. Science 353, aac9439 (2016).
17. Liu, Y. et al. Van der Waals heterostructures and devices. Nat. Rev. Mater. 1, 16042 (2016).

18. Liao, L. et al. High- $\kappa$ oxide nanoribbons as gate dielectrics for high mobility top-gated graphene transistors. Proc. Natl Acad. Sci. USA 107, 6711-6715 (2010).

This study first demonstrated vdW integration as a damage-free approach for graphene-dielectric integration with a pristine interface and retained electronic performance.

19. Liu, Y. et al. Approaching the Schottky-Mott limit in van der Waals metalsemiconductor junctions. Nature 557, 696-700 (2018).

This study generalized vdW integration for bond-free integration of 3D metal thin-film electrodes with 2D semiconductors to create nearly ideal metal/ semiconductor vdW junctions.

20. Wang, C. et al. Monolayer atomic crystal molecular superlattices. Nature 555, 231-236 (2018).

21. Dzyaloshinskii, I. E., Lifshitz, E. \& Pitaevskii, L. P. General theory of van der Waals forces. Phys. Uspekhi 4, 153-176 (1961).

22. Stone, A. The Theory of Intermolecular Forces (Oxford Univ. Press, Oxford, 2013).

23. Jones, J.E On the determination of molecular fields - - II. From the equation of state of a gas. Proc. R. Soc. A 106, 463-477 (1924).

24. Eskandari Nasrabad, A. \& Laghaei, R. Computational studies on thermodynamic properties, effective diameters, and free volume of argon using an ab initio potential. J. Chem. Phys. 125, 084510 (2006).

25. Autumn, K. et al. Evidence for van der Waals adhesion in gecko setae. Proc. Natl Acad. Sci. USA 99, 12252-12256 (2002).

26. Geim, A. K. et al. Microfabricated adhesive mimicking gecko foot-hair. Nat. Mater. 2, 461-463 (2003).

27. Wang, L. et al. One-dimensional electrical contact to a two-dimensional material. Science 342, 614-617 (2013).

28. Yue, R. et al. Nucleation and growth of $\mathrm{WSe}_{2}$ : enabling large grain transition metal dichalcogenides. 2D Mater. 4, 045019 (2017).

29. Fuhrer, M. et al. Crossed nanotube junctions. Science 288, 494-497 (2000).

30. Rueckes, T. et al. Carbon nanotube-based nonvolatile random access memory for molecular computing. Science 289, 94-97 (2000).

31. Duan, X., Huang, Y., Cui, Y., Wang, J. \& Lieber, C. M. Indium phosphide nanowires as building blocks for nanoscale electronic and optoelectronic devices. Nature 409, 66-69 (2001).

This paper presented the initial assembled semiconductor nanowire junctions that inspired a broad range of vdW-integrated nanowire electronics and optoelectronics.

32. Huang, Y. et al. Logic gates and computation from assembled nanowire building blocks. Science 294, 1313-1317 (2001).

33. Duan, X., Huang, Y., Agarwal, R. \& Lieber, C. M. Single-nanowire electrically driven lasers. Nature 421, 241-245 (2003).

34. Huang, Y., Duan, X. \& Lieber, C. M. Nanowires for integrated multicolor nanophotonics. Small 1, 142-147 (2005).

35. Novoselov, K. S. et al. Electric field effect in atomically thin carbon films. Science 306, 666-669 (2004).

36. Novoselov, K. et al. Two-dimensional atomic crystals. Proc. Natl Acad. Sci. USA 102, 10451-10453 (2005).

This work first demonstrated the exfoliation of various 2D crystals other than graphene.

37. Jariwala, D., Marks, T. J. \& Hersam, M. C. Mixed-dimensional van der Waals heterostructures. Nat. Mater. 16, 170-181 (2017).

38. Ponomarenko, L. et al. Tunable metal-insulator transition in double-layer graphene heterostructures. Nat. Phys. 7, 958-961 (2011)

39. Britnell, L. et al. Field-effect tunneling transistor based on vertical graphene heterostructures. Science 335, 947-950 (2012).

This study demonstrated the first vertical tunnelling transistor from 2D/2D vdW heterostructures.

40. Yang, H. et al. Graphene barristor, a triode device with a gate-controlled Schottky barrier. Science 336, 1140-1143 (2012).

41. Yu, W. J. et al. Vertically stacked multi-heterostructures of layered materials for logic transistors and complementary inverters. Nat. Mater. 12, 246-252 (2013).

This paper presented the first vertical logic integration from 2D vdW multi-heterostructures.

42. Yu, W. J. et al. Highly efficient gate-tunable photocurrent generation in vertical heterostructures of layered materials. Nat. Nanotechnol. 8, 952-958 (2013).

43. Roy, K. et al. Graphene-MoS 2 hybrid structures for multifunctional photoresponsive memory devices. Nat. Nanotechnol. 8, 826-830 (2013).

44. Britnell, L. et al. Strong light-matter interactions in heterostructures of atomically thin films. Science 340, 1311-1314 (2013).

45. Fang, H. et al. Strong interlayer coupling in van der Waals heterostructures built from single-layer chalcogenides. Proc. Natl Acad. Sci. USA 111, 6198-6202 (2014)

46. Lee, C.-H. et al. Atomically thin $\mathrm{p}-n$ junctions with van der Waals heterointerfaces. Nat. Nanotechnol. 9, 676-681 (2014).

47. Cheng, R. et al. Electroluminescence and photocurrent generation from atomically sharp WSe $\mathrm{S}_{2} / \mathrm{MoS}_{2}$ heterojunction p-n diodes. Nano Lett. 14, 5590-5597 (2014).

48. Yuan, H. et al. Polarization-sensitive broadband photodetector using a black phosphorus vertical p-n junction. Nat. Nanotechnol. 10, 707-713 (2015).

49. Withers, F. et al. Light-emitting diodes by band-structure engineering in van der Waals heterostructures. Nat. Mater. 14, 301-306 (2015). 
50. Ju, L. et al. Tunable excitons in bilayer graphene. Science 358, 907-910 (2017)

51. Xu, W. et al. Correlated fluorescence blinking in two-dimensional semiconductor heterostructures. Nature 541, 62-67 (2017).

52. Kang, K. et al. Layer-by-layer assembly of two-dimensional materials into wafer-scale heterostructures. Nature 550, 229-233 (2017). This paper presented the assembly of wafer-scale $2 \mathrm{D} \mathrm{vdW}$ heterostructures through layer-by-layer stacking.

53. Zhang, Z. et al. Robust epitaxial growth of two-dimensional heterostructures, multiheterostructures, and superlattices. Science 357, 788-792 (2017).

54. Mayorov, A. S. et al. Micrometer-scale ballistic transport in encapsulated graphene at room temperature. Nano Lett. 11, 2396-2399 (2011).

55. Lee, G.-H. et al. Flexible and transparent $\mathrm{MoS}_{2}$ field-effect transistors on hexagonal boron nitride-graphene heterostructures. ACS Nano 7, 7931-7936 (2013).

56. Cui, X. et al. Multi-terminal transport measurements of $\mathrm{MoS}_{2}$ using a van der Waals heterostructure device platform. Nat. Nanotechnol. 10, 534-540 (2015).

57. Li, L. et al. Quantum Hall effect in black phosphorus two-dimensional electron system. Nat. Nanotechnol. 11, 593-597 (2016).

58. Liu, Y. et al. Toward barrier free contact to molybdenum disulfide using graphene electrodes. Nano Lett. 15, 3030-3034 (2015).

59. Bandurin, D. A. et al. High electron mobility, quantum Hall effect and anomalous optical response in atomically thin InSe. Nat. Nanotechnol. 12 223-227 (2017)

60. Georgiou, T. et al. Vertical field-effect transistor based on graphene-WS heterostructures for flexible and transparent electronics. Nat. Nanotechnol. 8 , 100-103 (2013)

61. Hong, X. et al. Ultrafast charge transfer in atomically thin $\mathrm{MoS}_{2} / \mathrm{WS}_{2}$ heterostructures. Nat. Nanotechnol. 9, 682-686 (2014).

62. Rivera, P. et al. Observation of long-lived interlayer excitons in monolayer $\mathrm{MoSe}_{2}-\mathrm{WSe}_{2}$ heterostructures. Nat. Commun. 6, 6242 (2015).

63. Rivera, P. et al. Valley-polarized exciton dynamics in a 2D semiconductor heterostructure. Science 351, 688-691 (2016).

64. Unuchek, D. et al. Room-temperature electrical control of exciton flux in a van der Waals heterostructure. Nature 560, 340-344 (2018).

65. Gong, C. et al. Discovery of intrinsic ferromagnetism in two-dimensional van der Waals crystals. Nature 546, 265-269 (2017).

66. Huang, B. et al. Layer-dependent ferromagnetism in a van der Waals crystal down to the monolayer limit. Nature 546, 270-273 (2017).

The above two papers first reported 2D magnets, providing a new material platform for 2D magnets and magnetic vdW heterostructures

67. Huang, B. et al. Electrical control of 2D magnetism in bilayer $\mathrm{Crl}_{3}$. Nat Nanotechnol. 13, 544-548 (2018).

68. Klein, D. R. et al. Probing magnetism in 2D van der Waals crystalline insulators via electron tunneling. Science 360, 1218-1222 (2018).

69. Song, T. et al. Giant tunneling magnetoresistance in spin-filter van der Waals heterostructures. Science 360, 1214-1218 (2018).

70. Jiang, S., Li, L., Wang, Z., Mak, K. F. \& Shan, J. Controlling magnetism in $2 \mathrm{D} \mathrm{Crl}_{3}$ by electrostatic doping. Nat. Nanotechnol. 13, 549-553 (2018).

71. Seyler, K. et al. Valley manipulation by optically tuning the magnetic proximity effect in $\mathrm{WSe}_{2} / \mathrm{Crl}_{3}$ heterostructures. Nano Lett. 18, 3823-3828 (2018).

72. Cao, Y. et al. Correlated insulator behaviour at half-filling in magic-angle graphene superlattices. Nature 556, 80-84 (2018).

73. Cao, Y. et al. Unconventional superconductivity in magic-angle graphene superlattices. Nature 556, 43-50 (2018).

The above two papers first reported exotic electronic properties, including superconductivity arising in a twisted 2D/2D vdW homostructure.

74. Ni, Z. H. et al. Tunable stress and controlled thickness modification in graphene by annealing. ACS Nano 2, 1033-1039 (2008)

75. Liu, Y. et al. Plasmon resonance enhanced multicolour photodetection by graphene. Nat. Commun. 2, 579 (2011)

76. Konstantatos, G. et al. Hybrid graphene-quantum dot phototransistors with ultrahigh gain. Nat. Nanotechnol. 7, 363-368 (2012)

77. Liao, L. et al. Top-gated graphene nanoribbon transistors with ultrathin high-k dielectrics. Nano Lett. 10, 1917-1921 (2010).

78. Liao, L. et al. High-performance top-gated graphene-nanoribbon transistors using zirconium oxide nanowires as high-dielectric-constant gate dielectrics. Adv. Mater. 22, 1941-1945 (2010).

79. Liao, L. et al. High-speed graphene transistors with a self-aligned nanowire gate. Nature 467, 305-308 (2010).

This study first demonstrated damage-free vdW integration of 1D/2D heterostructures for creating a high-speed graphene transistor with a self-aligned nanowire gate.

80. Liao, L. et al. Sub-100 nm channel length graphene transistors. Nano Lett. 10, 3952-3956 (2010).

81. Cheng, R. et al. High-frequency self-aligned graphene transistors with transferred gate stacks. Proc. Natl Acad. Sci. USA 109, 11588-11592 (2012). This paper presented the first scalable vdW integration of a lithographically defined 1D gate array and 2D graphene with minimized interface damage to enable the construction of a high-speed transistor array.

82. Cheng, R. et al. Few-layer molybdenum disulfide transistors and circuits for high-speed flexible electronics. Nat. Commun. 5, 5143 (2014).

83. Jiang, S. et al. Real-time electrical detection of nitric oxide in biological systems with sub-nanomolar sensitivity. Nat. Commun. 4, 2225 (2013).

84. Jia, C. et al. Quantum interference mediated vertical molecular tunneling transistors. Sci. Adv. 4, eaat8237 (2018).

85. Bediako, D. K. et al. Heterointerface effects in the electrointercalation of van der Waals heterostructures. Nature 558, 425-429 (2018).
86. Sarkar, D. et al. A subthermionic tunnel field-effect transistor with an atomically thin channel. Nature 526, 91-95 (2015).

87. Liu, M. et al. A graphene-based broadband optical modulator. Nature $\mathbf{4 7 4}$ 64-67 (2011).

88. Yao, B. et al. Gate-tunable frequency combs in graphene-nitride microresonators. Nature 558, 410-414 (2018).

89. Popa, D. et al. Graphene Q-switched, tunable fiber laser. Appl. Phys. Lett. 98 , 073106 (2011)

90. Yao, B. et al. Graphene Q-switched distributed feedback fiber lasers with narrow linewidth approaching the transform limit. Opt. Express 25, 8202-8211 (2017).

91. Chow, C. M. et al. Unusual exciton-phonon interactions at van der Waals engineered interfaces. Nano Lett. 17, 1194-1199 (2017).

92. Lee, J. et al. Interfacial mode coupling as the origin of the enhancement of $T_{\mathrm{c}}$ in FeSe films on $\mathrm{SrTiO}_{3}$. Nature 515, 245-248 (2014).

93. Ahn, J.-H. et al. Heterogeneous three-dimensional electronics by use of printed semiconductor nanomaterials. Science 314, 1754-1757 (2006).

94. Kim, C., Burrows, P. E. \& Forrest, S. R. Micropatterning of organic electronic

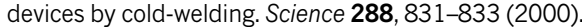

95. Meitl, M. A. et al. Transfer printing by kinetic control of adhesion to an elastomeric stamp. Nat. Mater. 5, 33-38 (2006).

96. Ding, S. et al. Reliable spin valves of conjugated polymer based on mechanically transferrable top electrodes. ACS Nano 12, 12657-12664 (2018).

97. Ko, H. et al. Ultrathin compound semiconductor on insulator layers for high-performance nanoscale transistors. Nature 468, 286-289 (2010),

98. Liu, Y. et al. Maximizing the current output in self-aligned graphene-InAsmetal vertical transistors. ACS Nano 13, 847-854 (2019).

99. Sun, Y., Choi, W. M., Jiang, H., Huang, Y. Y. \& Rogers, J. A. Controlled buckling of semiconductor nanoribbons for stretchable electronics. Nat. Nanotechnol. 1, 201-207 (2006).

100. Chen, C.-C., Chang, C.-C., Li, Z., Levi, A. \& Cronin, S. B. Gate tunable graphenesilicon Ohmic/Schottky contacts. Appl. Phys. Lett. 101, 223113 (2012).

101. Kioseoglou, G. et al. Electrical spin injection into Si: a comparison between $\mathrm{Fe} / \mathrm{Si}$ Schottky and $\mathrm{Fe} / \mathrm{Al}_{2} \mathrm{O}_{3}$ tunnel contacts. Appl. Phys. Lett. 94, 122106 (2009).

102. McCarthy, L. S. et al. GaN HBT: toward an RF device. IEEE Trans. Electron Dev. 48, 543-551 (2001).

103. Kyle, E. C. Kaun, S. W., Young, E. C. \& Speck, J. S. Increased p-type conductivity through use of an indium surfactant in the growth of Mg-doped GaN. Appl. Phys. Lett. 106, 222103 (2015).

104. Yim, K. H. et al. Efficient conjugated-polymer optoelectronic devices fabricated by thin-film transfer-printing technique. Adv. Funct. Mater. 18, 1012-1019 (2008).

105. Khang, D.-Y., Jiang, H., Huang, Y. \& Rogers, J. A. A stretchable form of single-crystal silicon for high-performance electronics on rubber substrates. Science 311, 208-212 (2006).

106. Tao, L. et al. Silicene field-effect transistors operating at room temperature. Nat. Nanotechnol. 10, 227-231 (2015).

107. Liu, Y., Duan, X., Huang, Y. \& Duan, X. Two-dimensional transistors beyond graphene and TMDCs. Chem. Soc. Rev. 47, 6388-6409 (2018).

108. Kretinin, A. et al. Electronic properties of graphene encapsulated with different two-dimensional atomic crystals. Nano Lett. 14, 3270-3276 (2014).

109. Kim, Y. et al. Remote epitaxy through graphene enables two-dimensional material-based layer transfer. Nature 544, 340-343 (2017).

110. Shim, J. et al. Controlled crack propagation for atomic precision handling of wafer-scale two-dimensional materials. Science 362, 665-670 (2018).

111. Duan, X. \& Lieber, C. M. General synthesis of compound semiconductor nanowires. Adv. Mater. 12, 298-302 (2000).

112. Xie, X.-L., Mai, Y.-W. \& Zhou, X.-P. Dispersion and alignment of carbon nanotubes in polymer matrix: a review. Mater. Sci. Eng. Rep. 49, 89-112 (2005).

113. Liao, L. et al. Scalable fabrication of self-aligned graphene transistors and circuits on glass. Nano Lett. 12, 2653-2657 (2011).

114. Tanase, M. et al. Magnetic alignment of fluorescent nanowires. Nano Lett. 1 , 155-158 (2001)

115. Geim, A. K. \& Novoselov, K. S. The rise of graphene. Nat. Mater. 6, 183-191 (2007).

116. Castellanos-Gomez, A. et al. Deterministic transfer of two-dimensional materials by all-dry viscoelastic stamping. 2D Mater. 1, 011002 (2014).

117. Gurarslan, A. et al. Surface-energy-assisted perfect transfer of centimeter-scale monolayer and few-layer $\mathrm{MoS}_{2}$ films onto arbitrary substrates. ACS Nano 8 , $11522-11528(2014)$

118. Cui, X. et al. Rolling up transition metal dichalcogenide nanoscrolls via one drop of ethanol. Nat. Commun. 9, 1301 (2018).

119. Desai, S. B. et al. Gold-mediated exfoliation of ultralarge optoelectronicallyperfect monolayers. Adv. Mater. 28, 4053-4058 (2016).

120. Zaretski, A. V. et al. Metal-assisted exfoliation (MAE): green, roll-to-roll compatible method for transferring graphene to flexible substrates. Nanotechnology 26, 045301 (2015).

121. Zaretski, A. V. \& Lipomi, D. J. Processes for non-destructive transfer of graphene: widening the bottleneck for industrial scale production. Nanoscale 7, 9963-9969 (2015)

122. Kim, J. et al. Layer-resolved graphene transfer via engineered strain layers. Science 342, 833-836 (2013).

123. Bae, S.-H. et al. Unveiling the carrier transport mechanism in epitaxial graphene for forming wafer-scale, single-domain graphene. Proc. Natl Acad. Sci. USA 114, 4082-4086 (2017). 
124. Li, X. et al. Large-area synthesis of high-quality and uniform graphene films on copper foils. Science 324, 1312-1314 (2009).

125. Lin, Z. et al. Solution processable colloidal nanoplates as building blocks for high-performance electronic thin films on flexible substrates. Nano Lett. 14 6547-6553 (2014).

126. Lin, Z. et al. Solution-processable 2D semiconductors for high-performance large-area electronics. Nature 562, 254-258 (2018).

127. Chen, G. \& Hui, P. Thermal conductivities of evaporated gold films on silicon and glass. Appl. Phys. Lett. 74, 2942-2944 (1999).

128. Sundar, V. C. et al. Elastomeric transistor stamps: reversible probing of charge transport in organic crystals. Science 303, 1644-1646 (2004).

129. Liu, C. et al. Improving solution-processed n-type organic field-effect transistors by transfer-printed metal/semiconductor and semiconductor/ semiconductor heterojunctions. Org. Electron. 15, 1884-1889 (2014).

Acknowledgements X.D. acknowledges support by the Office of Naval Research through grant number N00014-15-1-2368. Y.H. acknowledges support by the National Science Foundation through grant number EFRI-1433541.
Y.L. acknowledges support by Fundamental Research Funds for the Central Universities, China.

Reviewer information Nature thanks Jeehwan Kim and the other anonymous reviewer(s) for their contribution to the peer review of this work.

Author contributions All authors wrote and commented on the manuscript.

Competing interests The authors declare no competing interests.

Additional information

Reprints and permissions information is available at http://www.nature.com/ reprints.

Correspondence and requests for materials should be addressed to Y.H. or X.D. Publisher's note: Springer Nature remains neutral with regard to jurisdictional claims in published maps and institutional affiliations.

○ Springer Nature Limited 2019 